\title{
Wildfire safety with wireless sensor networks
}

\author{
Andrey Somov \\ CREATE-NET, via alla Cascata 56/D, 38123 Trento, Italy
}

\section{Abstract}

Nowadays, the Wireless Sensor Network (WSN) paradigm is extensively used for the environmental monitoring including wildfires. Like other disasters, this phenomenon, if not detected early, may have grave consequences, e.g. a significant pecuniary loss, or even lead to human victims. This paper surveys the approaches to early wildfire detection using WSN facilities with a special focus on real deployments and hardware prototypes. In our work we propose not merely a description, but a classification of the fire detection methods which are divided into three groups: gas sensing, sensing of environmental parameters, and video monitoring. Then the methods are comparatively analyzed from the viewpoints of the cost, power consumption, and implementation complexity. Finally, we summarize our vision of the prospects of resolving the wildfire detection problem using WSNs.

Keywords: environmental monitoring, remote sensing, sensor node, wildfire, wireless sensor network

Received on 1 July 2011; revised version received on 20 September 2011; accepted on 20 September 2011

Copyright (c) 2011 Somov, licensed to ICST. This is an open access article distributed under the terms of the Creative Commons Attribution licence (http://creativecommons.org/licenses/by/3.0/), which permits unlimited use, distribution and reproduction in any medium so long as the original work is properly cited.

doi: 10.4108/trans.amsys.2011.e4

\section{Introduction}

The use of sensor networks covering large territories to ensure the effective monitoring of such phenomena as wildfires remains to be a problem of particular interest and significance [1]. Wildfire (see Figure 1), being an uncontrolled fire, destroys vegetation (forests, bushes, grass), animal species and spreads quickly over large areas, therefore posing a threat to human health, lives, and livelihoods (see Table 1). Wildfire can be defined as both a natural disaster resultant from the hot weather conditions or, for example, lightning and a man-caused phenomenon.

There are currently a number of approaches to address this problem. However, from a technical point of view, it has not been completely and effectively resolved as yet [2].

Traditionally, the monitoring task was performed by a specially trained team in a lookout tower located at a high point [3]. This method of monitoring is still applicable in some countries, such as the US, Canada, and Australia. Due to unreliability of human observations, some vision techniques have been proposed to monitor small forests from the tower [4].
Satellite imaging, for example, though able to ensure sensing of vast areas, has, as a method, considerable restraints in terms of real-time spatial resolution and sensitivity. In addition, it is associated with the exceptionally high deployment and operational costs, which make it difficult to use in all cases $[5,6]$.

Another widely applied method of monitoring wildfires or gas leakages is based on getting data on the emissions source. The ultrasensitive devices installed aboard the airplanes, trailers, and other vehicles enable the acquisition of data in cross patterns [2]. This approach requires the availability of operators and maintenance personnel. Its main shortcoming, however, is that spatial and time resolution is limited to a point measurement at the vehicle current location. Apart from that, in the case of using airborne platforms their ultrahigh sensitivity should be provided to detect ground emissions after the gases have propagated to a considerable distance from the sources.

In order to simplify and reduce the costs of fire monitoring, the concept of Wireless Sensor Networks (WSNs) [7] has been recently proposed. Cheap and compact wireless sensor devices deployed over a large territory and operating both jointly and autonomously may be effectively used to detect hazardous gases and monitor wildfires [8]. 


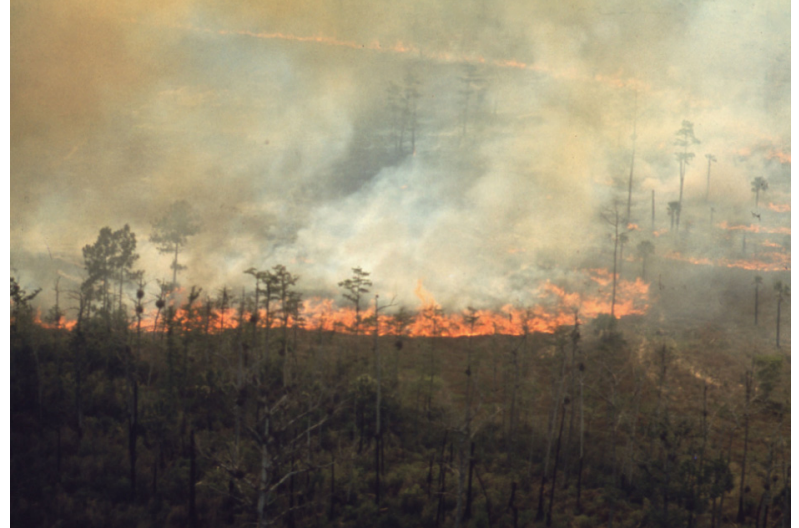

Figure 1. Fire in the Everglades National Park, USA (photograph: Flip Schulke).

In the context of wildfire, WSN should satisfy the following requirements:

- Low cost. The monitoring of wild lands suggests the availability of many nodes spread over the surveyed area. As there always is a risk of losing the nodes during the fire, the cost of a sensor node should be minimal.

- WSN lifetime. Since the WSNs for wildfire monitoring are deployed in difficult-to-access areas, the power source has to support the long-term operation of a sensor node.

- Early fire detection. Since the wildfire spreads quickly, the WSN has to fast and accurately detect or predict the fire and send an alarm signal to the firefighters as soon as possible.

The current advances in ICT successfully address the Low-cost and WSN lifetime requirements. The typical off-the-shelf electronic components available on the market are cheap. The lifetime problem in the WSN domain is addressed from several viewpoints, i.e. duty-cycle adjustment [9], power management [10], WSN synchronization [11], and energy scavenging [12]. The Early fire detection requirement makes us carefully consider the fire phenomena and their progress in time. Figure 2 shows a generic diagram of the fire formation. We shall see later how important this diagram is for the fire detection and WSN development.

As it was mentioned above, the fire starts with overheating, as a result of hot weather conditions or lightning. Due to overheating or smouldering of wood materials, the pyrolysis products, primarily carbon oxide (CO) and hydrogen $\left(\mathrm{H}_{2}\right)$, evolve in the atmosphere. Finally, smoke and flame appear.

The present paper aims at surveying the existing approaches to and sensor systems for the wildfire monitoring and detection. We classify these systems into three groups based on the wildfire detection approach used and offer a comparative analysis of the systems.

The paper is organized as follows: Section 2 will briefly introduce the reader to the WSN architecture and then will overview the related environmental applications of WSNs. A survey of existing sensor systems for wildfire detection and their classification are presented in Section 3. Section 4 provides the comparative analysis of these systems. Finally, in Conclusion we summarize our vision of the prospects of resolving the problem.

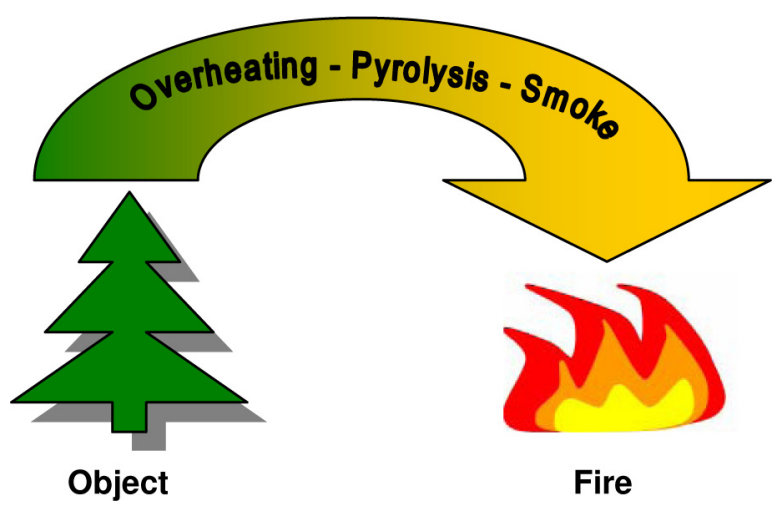

Figure 2. Generic fire formation diagram.

Table 1. Average area of forest annually affected by fire by region, 2005 [35].

\begin{tabular}{|c|c|c|c|}
\hline \multirow[t]{2}{*}{ Region } & \multicolumn{2}{|c|}{ Information availability } & \multirow{2}{*}{$\begin{array}{c}\text { Area of forest } \\
\text { affected by fire } \\
\text { (\% of forest area) }\end{array}$} \\
\hline & $\begin{array}{l}\text { Number of } \\
\text { countries }\end{array}$ & $\begin{array}{l}\% \text { of total } \\
\text { forest area }\end{array}$ & \\
\hline Africa & 21 & 22.4 & 5.4 \\
\hline Asia & 29 & 87.9 & 0.5 \\
\hline Europe & 42 & 99.4 & 0.1 \\
\hline North and Central America & 15 & 98.9 & 0.5 \\
\hline South America & 5 & 14.0 & 0.3 \\
\hline World & 118 & 65.2 & 0.7 \\
\hline
\end{tabular}




\section{Environmental WSN}

The history of the environmental monitoring is long enough [13]. The WSNs used for this application are deployed in potentially harsh and remote environments where the inevitable node and communication failures have to be tolerated. It means that apart from the WSN lifetime requirement presented in the Introduction, there are two other significant challenges associated with the environmental monitoring application:

- Radio communication. Radio chip is generally the most 'hungry' component on board [14]. The energy spent for communication and used to set up ad hoc networks and clusters should therefore be minimized. Besides, radio communication in remote areas is characterized by unpredictability in wet and windy conditions.

- Remote management. The impossibility of a regular examination of systems in isolated regions makes remote access a mandatory requirement. Bugs are to be fixed, subsystems might need to be shut down, and schedules changed.

A sensor network (see Figure 3) is designed to ensure data transmission from an array of sensors to a user's data repository on a server. They do not necessarily use a simple one-way data stream over a communications network. The system components are able to make decisions as to what data are to be transmitted in order to minimize power use while maximizing the information content.

Proceeding from sensor nodes toward the user the systems, e.g. sink, gate, usually build up the computer power, data storage, and power availability. If a large number of sensor nodes are available for one gate (network coordinator), they are normally arranged as an ad hoc set of clusters with representative nodes (sinks) communicating a group's data to the gate.

A WSN consists of wireless nodes which measure physical conditions (i.e. temperature, humidity, pressure) using sensors, digitize the measurements, and store or distribute the measured data over the network. The sensor nodes are connected by a radio channel. A typical wireless sensor node block diagram is presented in Figure 4.

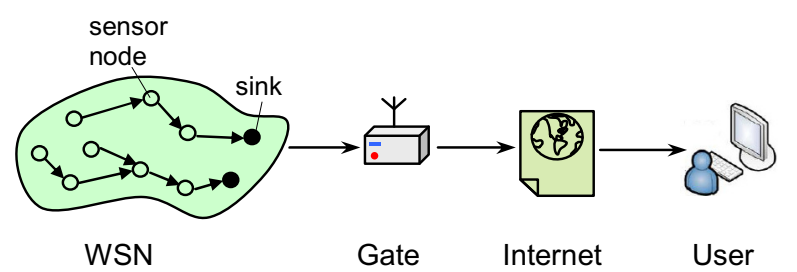

Figure 3. Typical sensor network architecture.

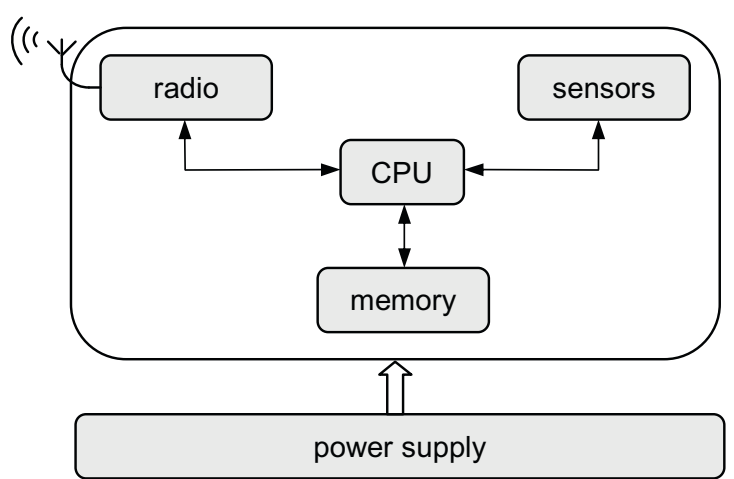

Figure 4. Architecture of a typical wireless sensor node.

A typical wireless sensor node has five main blocks: (i) Central Processing Unit (CPU), (ii) memory chip, (iii) radio transceiver, (iv) one or more sensors, and (v) power supply.

Due to low power consumption and good performance, a Microcontroller Unit (MCU) typically serves as CPU in a sensor node. It controls the data acquisition process, synchronizes the components on board and communication with other nodes. Communication within the network is usually organized using the IEEE 802.15.4 standard [15], while data are transmitted over the ZigBee protocol stack [16]. Among the known data wireless transmission standards, such as Bluetooth, WiFi, etc., ZigBee appears to be the most acceptable in terms of the trade-off between the bandwidth and power consumption. Depending on the application, a sensor node may have various extra hardware components on board. Power supply of a sensor node is implemented as a (rechargeable) battery. However, in order to ensure long autonomous operation, some sensor node platforms also adapt super capacitors [17] and/or energy scavenging technology [18].

The following examples show the WSN application in environmental monitoring.

ZebraNet [19] is a GPS-based WSN to track the animal migration with sensors built in collars on the necks of zebras. The wireless sensor node has the following HW features: a single energy buffer as a lithium-ion battery, a dual-clock microcontroller, separate power supplies for each device to lower energy consumption, and an on-chip memory since the system has to generate detailed logs. The middleware of the system has GPS sensing and radio communication as priority events, and the remaining events could be regarded as a combination of scheduled and unplanned events. In order to reduce power consumption, the developers have carried out three low-level techniques: the timely use of components, the on-the-fly processing, and the dual-clock scheme which helps consume twice less energy using a slow clock. Therefore, two main technical problems tackled in this scenario are lifetime and localization of sensor nodes. 
A study of an active volcano is presented in [20]. The WSN consisting of 16 Tmote Sky sensor nodes was deployed on the Reventador volcano in Northern Ecuador. The study required high data rates and high data fidelity. The nodes were equipped with a microphone and a seismometer to collect the seismic and acoustic data on volcanic activity. These sensors were hosted on a separate acquisition board. Two D-type batteries powered each sensor node. The batteries within the entire network were changed twice during its 3 -week deployment. The radio link between the sensor network and the observatory point was supported by three modems. Batteries and photovoltaic cells powered the modems.

The glacier dynamics in Iceland is analyzed in [21]. This deployment aims to develop the improved models of the glacier behavior. The network is of a star configuration. The PIC18 MCU-based sensor nodes are supplied by three $3.6 \mathrm{~V}$ batteries wired in parallel. The custom design of the antenna allows transmission through the ice. Some of the probes are placed within the ice, while most of them are located $10-30 \mathrm{~cm}$ below. The probes collected data once per hour. The gateway is an ARMbased platform. In order to decrease the high sleep current of the CPU, the MSP430 MCU was used which put ARM to the power-off mode and upon request powered it up. For the long-term operation the gateway supports solar and wind energy harvesting. The gateway is also equipped with a GPS antenna for logging the glacier dynamics and a GPRS antenna to transmit the collected data.

A global shift of flora from the grass to tree vegetation is addressed in [22]. Light Under Shrub Thicket for Environmental Research (LUSTER) system deployed under the shrubs in Hog Island, USA, studies the effects of sunlight to evaluate the light dynamics in the context of ecological research. LUSTER's architecture is composed of a sensor node layer, a storage layer, and a delay-tolerant networking layer. The custom design of the sensor node is compatible with Mica2 and MicaZ motes. In fact, two versions of the node were developed. The first one, called 'Medusa', is the eight-channel node with light sensors. 'Solar Dust' has solar cells instead. This approach allows the harvesting of solar energy which is stored either in the super capacitor or in the battery. Voltage generated by solar cells is also assessed in terms of light intensity.

The present paper does not aim at describing all the available environmental applications of WSN. More detailed information on the subject can be found in $[23,24]$. However, it should be noted that all these systems have encountered the challenges of being deployed in remote areas with limited access and power resources. The lifetime challenge appears to be a common problem associated with all the environmental WSNs listed above.

In the next section we are focusing our attention on wildfire monitoring using WSN.

\section{Wildfire monitoring with WSN}

The wildfire detection WSN should detect the fire at an early stage (see diagram in Figure 2). In this section we classify the currently available fire detection approaches into three groups:

(i) Gas sensing. This approach helps detect the fire at the pyrolysis phase. Wood pyrolysis is accompanied by the $\mathrm{H}_{2}$ and $\mathrm{CO}$ emission in the atmosphere. For the atmosphere analysis a wireless node with a specific sensor on board is applied.

(ii) Environmental parameters' sensing. The sensor nodes measure the ambient temperature, humidity, light, and barometric pressure to detect the fire.

(iii) Video monitoring. In this case the WSN is a mix of sensor nodes and video cameras which are used to verify an alarm received from the sensor nodes.

The more detailed analysis of the three approaches is presented below.

\subsection{Gas sensing}

This approach is based on the periodical sensing of the atmosphere with the purpose of discovering a specific gas which precedes the smoke and inflammation. This strategy helps predict the fire at an early stage. However, the sensor nodes which adapt this technology are power 'hungry' devices and they are quite expensive. Besides, the presented platforms of sensor nodes have not been deployed in real conditions as yet.

Semiconductor sensor. An autonomous wireless sensor system for early fire detection is presented in [17]. This system consists of sensing and power management modules. The sensing module has an on-board semiconductor sensor which can detect the pyrolysis products $\left(\mathrm{H}_{2}, \mathrm{CO}\right)$ and, therefore, detect the fire before inflammation. The principle of its operation is based on changing the sensor's sensitive layer conductivity in response to gas absorption (the conductivity increases as the gas concentration gets higher). The physical and chemical processes on the sensitive layer's surface are activated by heating, which increases its sensitivity to certain gases.

The power management module is a device which supports the energy scavenging technology. It can accumulate energy both from alternating and direct current based harvesters, though not at the same time. The harvested energy is stored in a battery and/or two super capacitors wired in series. The experimental system deployment shows that it takes around $6 \mathrm{~s}$ for the environment sensing and data communication (issuing an alarm signal) to the host node. The alarm signal is being generated upon exceeding the pyrolysis density threshold in the atmosphere. The application of the energy scavenging 
Table 2. Power consumption for some off-the-shelf sensors used for fire detection.

\begin{tabular}{lll}
\hline Sensor (manufacturer) & Sensing parameter & Power consumption, mW \\
\hline MS5540B (Intersema) & Barometric pressure & 0.012 \\
STDS75 (STM) & Temperature & 0.5 \\
HIH-4000-001 (Honeywell) & Humidity & 10 \\
EC-5 (Decagon) & Soil moisture & 30 \\
SGS-2140, semiconductor (Delta-S) & $\mathrm{CO}, \mathrm{H}_{2}$ & 215 (pulse heating) \\
MQ-4 semiconductor (Hanwei Electronics) & Smoke & 750 \\
GGS-2000T (UST) & CO, $\mathrm{H}_{2}$ & 800 (constant heating) \\
Spectroscopy sensor [25] & Dangerous gases & $800 \mathrm{~mA}$ \\
\hline
\end{tabular}

technology based on photovoltaic harvesting supports the stable operation of the system. However, the power consumption of the semiconductor sensor SGS-2140 (see Table 2) can be a limiting factor for the network deployment in sunless areas due to unavailability of other energy scavenging technologies providing a sufficient amount of energy for the network operation [18].

Spectroscopy sensor. The LaserSPECks sensor node platform [25] is developed for gas sensing applications using quartz-enhanced photoacoustic spectroscopy. This method operates as follows: the sensor checks whether an air sample is optically clear. If not, it means that the sample contains any other contaminant material. Due to specific molecular structures, each contaminant has the exact amount of light absorption associated with it. As compared to semiconductor sensors, the optical approach is much more reliable for detecting a specific gas.

The hardware architecture of LaserSPECks vl.0 includes two MCUs. The first one performs either measurements or communication while the other one calculates the sampled data. However, the sensor consumes up to $800 \mathrm{~mA}$, which is a significant disadvantage of the system. The platform is implemented using off-the-shelf components and is based on the Generalized Network of Miniature Environmental Sensors (GNOMES) 3.0 platform [26]. The LaserSPECks's developers claim that the second version of the platform will consume less power to simplify its deployments in remote areas.

\subsection{Sensing of environmental parameters}

A WSN which supports multiple sensors for measuring the environmental conditions is the most popular approach to fire detection. This is primarily due to the fact that the temperature, humidity, barometric pressure, and soil moisture sensors have low power consumption (see Table 2). This feature, along with an appropriate duty cycle, may provide an opportunity for the WSN of this kind to operate for several years.

While monitoring almost the same environmental parameters, these WSNs alarm their users following different techniques which are described below.

Calculation of 'forest-fire risk-level' index. The Forest-Fire Surveillance System (FFSS) performs the real-time moni- toring of the mountains in South Korea [27]. The FFSS uses TIP50CM off-the-shelf motes [28] which integrate the temperature, humidity, and light sensors. The nodes support the TinyOS operating system. The data collected by the sensor nodes are then transmitted to the gateway through the sink nodes. The gateway, connected to Internet, performs the preliminary verification and analysis of the received packets and in the case of their correctness forwards the data to FFSS. Upon receiving the data, a middleware program calculates the forest-fire risk-level index defined by the Office of Forestry. If the index corresponds to the fire danger level, the FFSS automatically alarms the user and the system administrator.

Threshold rule. A WSN system for the early forest fire detection with special focus on low power consumption is proposed in [29]. The designed system measures different ambient parameters at different heights of the trees. To ensure the early fire detection, the environmental temperature, relative humidity, barometric pressure, light intensity, smoke, and soil moisture are measured and processed by the sensor node. All the sensors except for the smoke one are off-the-shelf components. The fire alarm process starts with the detection of smoke. Other sensors on the node keep monitoring the environment. As soon as the risk threshold for each of the sensors is exceeded, the node generates a warning message.

Each custom sensor node is powered by two AA batteries. The authors claim at least 1 year of the system operation, which is achieved by the energy-efficient hardware, data acquisition, signal processing, and communication. This system has not been deployed in real conditions as yet. It is supposed, however, that its user will be able to remotely update the status of the ambient conditions. Besides, with the GSM/GPRS technology on a network coordinator, the response can be received by mobile communications.

Another example of using the 'threshold rule' to warn the user is the 'FireBug' WSN described in [1]. The WSN based on Mica2 motes with the independently mounted MTS420CA sensor board was deployed in California, USA. The sensor board hosts the temperature, relative humidity, barometric pressure, acceleration, light intensity, and GPS location sensors. The light intensity and acceleration sensors were not used during the field 
testing. The GPS unit was only needed for an accurate location of the mote. Once it has been defined, the GPS unit is turned off. The main purpose of this WSN is to investigate the system's ability to detect the fire and the robustness of the hardware in wildfire conditions. The WSN managed to record the flame passage. The nodes within this deployment were scorched and the fire was put out at the final stage. However, before being destroyed, the nodes reported the temperature increase and the barometric pressure and humidity decrease as the flame front advanced.

The forest fires on the Eastern hills in Bogota, Columbia, happen frequently. In order to address this problem, the forest fire early detection system using WSN is proposed in [30]. The WSN is deployed using off-the-shelf devices from Crossbow: MDA300 acquisition board, Mica2 mote, MIB520 gateway. The MoteView software helps in the WSN configuration and capturing of temperature and humidity. Besides, the software allows a user to define the thresholds for both environmental parameters. The system, however, generates an alarm signal after the temperature threshold has been exceeded.

A WSN for wildfire monitoring with the capability of adjusting the sensing parameters in accordance with the weather conditions is presented in [31]. Similar to the sensor nodes presented in [1] and [30], this WSN adopts off-the-shelf sensor node and acquisition sensor board (Mica2 and MTS400, respectively). The acquisition board hosts typical sensors, i.e. temperature, relative humidity, light intensity, barometric pressure, acceleration, and GPS location. The collected data are forwarded from the sensor node to the base station through the routing nodes. The base station processes the data and stores them in the database connected to the web server. The client's PC queries the web server and provides the user with the latest information and location on the captured data. In order to ensure the long-term operation of the WSN, the nodes perform sensing in the sunny and dry weather conditions (when the fire probability is higher) with a high duty cycle. On the contrary, at night time or when raining the nodes collect the data with a low duty cycle. If the fire is detected by one of the nodes, the adjacent nodes start sensing the environment with the increased duty cycle.

\subsection{Video monitoring}

Apart from the sensor nodes with typical sensors for monitoring the environmental conditions, a WSN may include some video cameras which ensure the exact location of the (potential) fire.

The fires destroy large areas of wild lands in Spain. To ensure the fire early detection and verification, the WSN deployment which contains multi-sensor nodes and video cameras is proposed in [32]. The authors use Linksys WRT54GL router as a sensor node supporting the IEEE
$802.11 \mathrm{~b} / \mathrm{g}$ wireless interface. The fire infrared and smoke detectors are connected through serial ports. These detectors are used for the environment sensing and fire alarming. In fact, an alarm message is only generated when both sensors have values higher than a threshold. Upon receiving an alarm message, the central server selects the appropriate video camera and transmits video information to the firefighters in order to verify the alarm signal. In order to support the WSN long-term operation, the energy scavenging technology is applied. A large number of sunny days in Spain allow using the photovoltaic cells. The preliminary calculations show that the photovoltaic system can support the stable operation of the WSN without any difficulties.

The WSN developed in [33] deals with the monitoring of weather conditions, e.g. temperature, humidity, thermal belts, and temperature inversions, in the wildland fire environments. This deployment consists of three WSNs and two video cameras. The cameras were set up at Hells Half Acre and Spot Mountain. The WSNs were set up at Hells Half Acre (six nodes), Kit Carson (five nodes), and Spot Mountain (two nodes) in the USA. The deployment is a multi-tiered portable system called 'FireWxNet' which comprises two parts. The aim of the first part is to bring communications to the remote areas where sensing will be performed. The second part is a multi-hop WSN which includes two video cameras with an autonomous power source. The sensor nodes in the network are made of a modified off-the-shelf Mica2 platform [34]. Each node is equipped with a temperature sensor, an accurate relative humidity sensor, and an anemometer for the wind direction and speed measurement. In the daytime the WSN operates using solar energy, at night it uses batteries. The main purpose of this system, however, is studying the fire behavior to give the firefighting community the opportunity of measuring and viewing the fire and weather conditions easily and safely.

\section{Comparative analysis}

Table 3 summarizes the available approaches to wildfire monitoring and sensor node platforms used for this application. However, only three out of nine WSNs were deployed in real conditions.

Gas sensing approach seems the most reliable and accurate since it predicts the fire before inflammation by detecting the pyrolysis products in the atmosphere. However, each platform hosts the sensors which consume too much power: semiconductor and spectroscopy ones. Besides, the cost of the semiconductor sensor used in [17] is of the order of $\$ 30$ (US); LaserSPECks node costs around $\$ 2 \mathrm{k}$ (US). The developers of LaserSPECks node claim that the price per node can be less than $\$ 100$ (US) with suitable advances in laser manufacturing and increase in the application demand. The developers of the system based on the semiconductor sensor are intending to 
TI Table 3. WSN platforms for wildfire monitoring.

\begin{tabular}{|c|c|c|c|c|c|c|}
\hline & System & Application & Sensor node platform & Real deployment & Advantages & Drawbacks \\
\hline \multirow[t]{2}{*}{ Gas sensing } & $\begin{array}{l}\text { Autonomous } \\
\text { system for early } \\
\text { fire detection [17] }\end{array}$ & $\begin{array}{l}\text { Pyrolysis } \\
\text { detection }\end{array}$ & $\begin{array}{l}\text { Custom node } \\
\text { Sensor: } \\
\text { semiconductor } \\
\left(\mathrm{CO}, \mathrm{H}_{2}\right)\end{array}$ & $\begin{array}{l}\text { No (only test } \\
\text { deployment) }\end{array}$ & $\begin{array}{l}\text { - the system uses } \\
\text { PV energy } \\
\text { - fire prediction } \\
\text { before inflammation }\end{array}$ & $\begin{array}{r}- \text { high price } \\
\text { per sensor }\end{array}$ \\
\hline & LaserSPECks [25] & $\begin{array}{l}\text { Dangerous gas } \\
\text { detection }\end{array}$ & GNOMES platform & No & $\begin{array}{l}\text { - accurate and early } \\
\text { fire prediction }\end{array}$ & $\begin{array}{l}- \text { high power } \\
\text { consumption }\end{array}$ \\
\hline \multirow[t]{5}{*}{$\begin{array}{l}\text { Sensing of } \\
\text { environmental } \\
\text { parameters }\end{array}$} & FFSS [27] & $\begin{array}{l}\text { Forest fire } \\
\text { monitoring } \\
\text { in South Korea }\end{array}$ & $\begin{array}{l}\text { TIP50CM mote } \\
\text { from Maxfor }\end{array}$ & No & $\begin{array}{l}\text { - calculates the } \\
\text { forest-fire risk-level } \\
\text { index for alarming }\end{array}$ & $\mathrm{N} / \mathrm{A}$ \\
\hline & $\begin{array}{l}\text { WSN for early } \\
\text { forest fire } \\
\text { detection [29] }\end{array}$ & $\begin{array}{l}\text { Forest fire } \\
\text { detection }\end{array}$ & $\begin{array}{l}\text { Custom node Sensors: } \\
\text { temperature, humidity, } \\
\text { barometric pressure, } \\
\text { light intensity, smoke, } \\
\text { soil moisture }\end{array}$ & No & 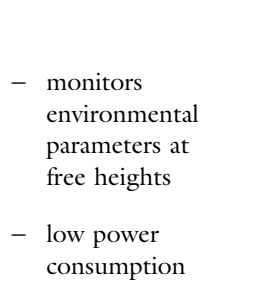 & $\mathrm{N} / \mathrm{A}$ \\
\hline & FireBug [1] & $\begin{array}{l}\text { Wildfire } \\
\text { monitoring in } \\
\text { California, USA }\end{array}$ & $\begin{array}{l}\text { Mica2 mote and } \\
\text { MTS420CA } \\
\text { sensor board } \\
\text { Sensors: temperature, } \\
\text { relative humidity, } \\
\text { barometric pressure }\end{array}$ & Yes & $\begin{array}{l}\text { - the research } \\
\text { community } \\
\text { gained the } \\
\text { experience in real } \\
\text { deployment }\end{array}$ & $\begin{array}{l}\text { - did not really } \\
\text { help the } \\
\text { firefighters }\end{array}$ \\
\hline & $\begin{array}{l}\text { System for early } \\
\text { forest fire } \\
\text { detection [30] }\end{array}$ & $\begin{array}{l}\text { Fire monitoring } \\
\text { at the Eastern } \\
\text { hills in Bogota, } \\
\text { Columbia }\end{array}$ & $\begin{array}{l}\text { Mica2 mote and } \\
\text { MDA300 } \\
\text { sensor board } \\
\text { Sensors: } \\
\text { temperature, } \\
\text { humidity }\end{array}$ & No & $\begin{array}{l}\text { - the system is } \\
\text { based on } \\
\text { hardware and } \\
\text { software from } \\
\text { a single vendor }\end{array}$ & $\begin{array}{l}\text { - alarming } \\
\text { when the } \\
\text { temperature } \\
\text { threshold is } \\
\text { exceeded }\end{array}$ \\
\hline & $\begin{array}{l}\text { WSN for wildfire } \\
\text { monitoring [31] }\end{array}$ & $\begin{array}{l}\text { Wildfire } \\
\text { monitoring }\end{array}$ & $\begin{array}{l}\text { Mica2 mote and } \\
\text { MDA400 } \\
\text { sensor board } \\
\text { Sensors: } \\
\text { temperature, humidity, } \\
\text { barometric pressure, } \\
\text { light intensity }\end{array}$ & No & $\begin{array}{l}\text { - the system } \\
\text { adjusts sensing } \\
\text { duty cycle in } \\
\text { accordance } \\
\text { with the weather } \\
\text { conditions }\end{array}$ & $\mathrm{N} / \mathrm{A}$ \\
\hline
\end{tabular}




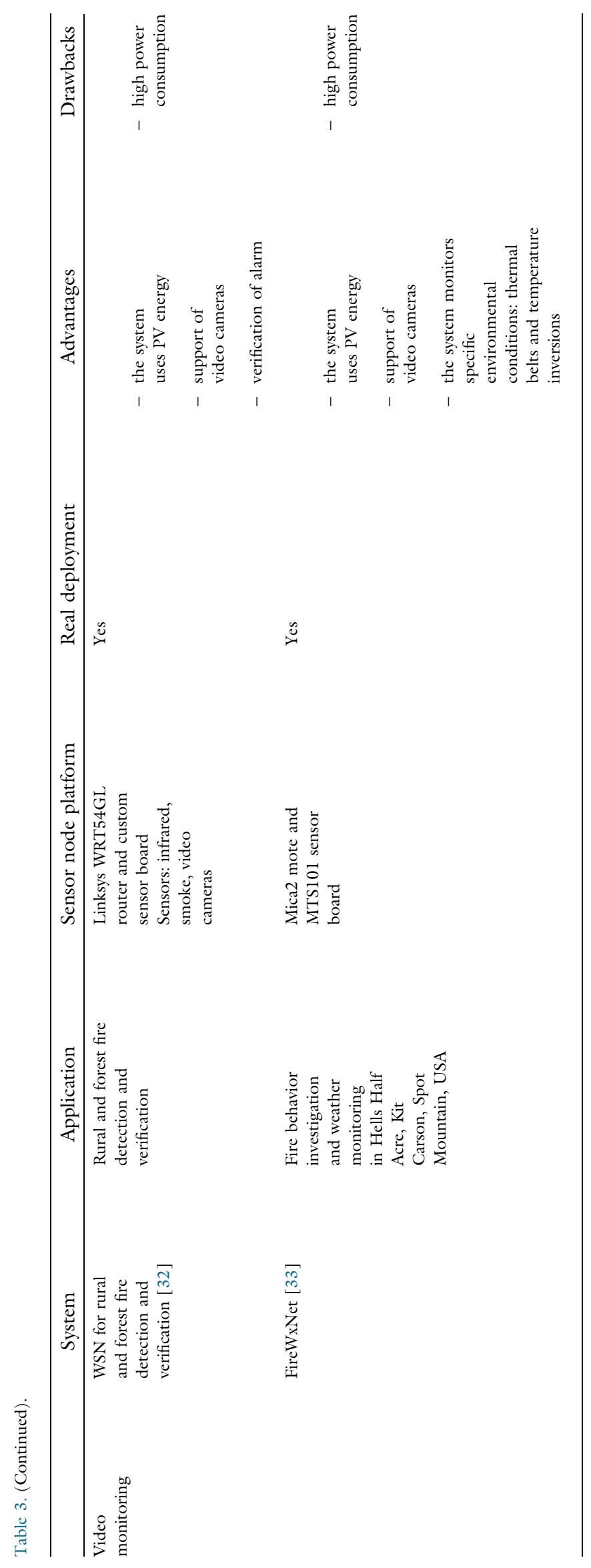

deploy the network in real conditions by applying the energy scavenging technology and thanks to lower price in comparison with the LaserSPECks platform. In fact, the high price and power consumption can be the limiting factors for the LaserSPECks network experimentation in the field.

Among the three approaches analyzed in the present work the technique based on sensing the environmental parameters appears to be the simplest, cheapest, and most energy efficient. The sensor nodes currently available on the market [34] either have some typical (temperature, humidity) sensors on board or can easily expand their sensing capabilities by using a sensor acquisition board [34]. Besides, these sensors, except for the smoke one, consume a negligible amount of power (see Table 1), which allows the nodes to operate for more than 1 year [29]. However, this approach can detect (not predict like gas sensing platforms) the fire at an early stage, i.e. when the direct flame has already appeared. Since the wildfire spreads quickly, the WSN of this kind cannot really help to put out the fire [1], but can be useful for research purposes to analyze the fire behavior and environment during the fire.

Video monitoring seems to be a trade-off between the two previous approaches. It is a power 'hungry' approach, but the application of the energy scavenging technology (PV energy) can help to resolve this problem. Smoke and infrared sensors are used for fire alarming [32], while video cameras are to verify the alarm signal. The video facilities, however, can be used for time-to-time monitoring [33]. It should also be noted that one camera can cooperate with several sensor nodes.

The experience gained during the WSN deployment in real conditions $[1,32,33]$ shows that the user must be alarmed very quickly, i.e. potential fire should be detected at an early stage. The network topology therefore should be simple and reliable enough so as not to add any delays in the alarm signal propagation. The network topologies proposed in [27] and [31], for example, do not meet this requirement having two 'mediator' tiers between the sensor node and the user.

Figure 5 presents a typical network communication scheme for the wildfire monitoring applications.

The sensor nodes usually support IEEE 802.15.4 standard and transmit data using ZigBee protocol stack (see Section 2). It is obvious that the network coordinator should support two communication technologies (which do not interfere with each other): one to communicate with the nodes and the other one to forward data from the nodes to the user. The system described in [29] proposes a combination of ZigBee and GSM/GPRS communications. These communication facilities do not interfere and allow the receiving of an alarm by mobile communication.

In our vision the prospects of using WSN for wildfire monitoring are in adopting useful features of all applicable 


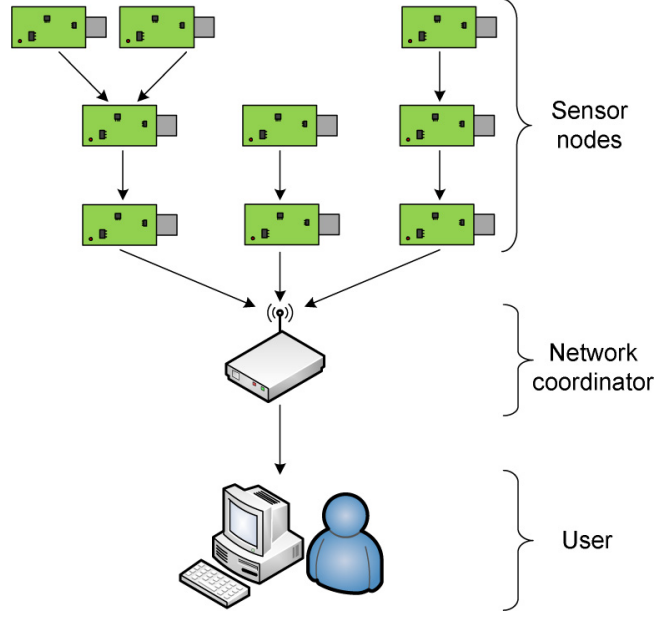

Figure 5. WSN communication scheme for wildfire monitoring application.

approaches, i.e. early fire formation prediction (gas sensing), low cost and fast prototyping of the WSN (sensing of environmental parameters), and fire verification (video monitoring). However, from the viewpoint of power consumption such an integration remains to be problematic.

\subsection{Comparison of deployed WSNS}

We start the comparison of deployed WSNs by defining their objectives which, in fact, are different. FireBug [1] (classified as sensing of environmental parameters) is mainly focused on engineering aspects of deployment and deployed by the specialists with technical background. In contrast, FireWxNet [33] and WSN for rural and forest fire detection [32] (classified as video monitoring) are deployed by the specialists with different research background. It should be noted, however, that video monitoring networks have different objectives. FireWxNet is focused on fire behavior investigation and weather monitoring. WSN for rural fire detection aims at fire detection and verification.

The networks we compare are designed using off-theshelf sensor nodes and base stations/routers. Due to the application of video cameras both video monitoring WSNs are characterized by high power consumption. In order to address the problem of long-term operation, both networks apply the energy scavenging technology. FireBug is powered by the batteries only.

The communication scheme (from the sensors to the user) in FireBug is organized with the following principles. The data from motes (operate on $433 \mathrm{MHz}$ frequency) are first collected by base station and then forwarded to the user through a server. This WSN communication scheme is both simple and reliable.

Due to its deployment features, FireWxNet uses more complicated data delivery to the user. A number of sensor nodes are wirelessly connected to the base station (IEEE802.11) through the sensor gate. The sensor gate is the sensor node connected to the base station using USB cable. The video camera can also be connected to the base station by wired means. The base stations with the directional antennas form the network backhaul tier. A satellite dish (connected to one of the base stations by wires) located in the researchers' camp provides the backhaul with the Internet access. Upon receiving an alert message a network operator in the camp can easily forward it to a special rescue service.

WSN for rural fire detection uses similar to FireBug's communication scheme: the sensor nodes and video cameras are connected to the access points (IEEE802.11g) which forward the data to the user. However, the video cameras are used for the verification of alert message from the sensor node. As soon as the possibility of fire is detected by the sensors, the network operator turns on the respective video camera which covers this 'alert' zone and checks if the received alert is true.

The WSN for rural fire detection seems the most successful system out of totally three deployments presented in this survey. This network provides secure fire monitoring with the fire verification option which can help to avoid false detections. Besides, the rare usage of the video cameras (for verification purposes) as well as photovoltaic system for the battery replenishment provides potentially long lifetime cycle for the system.

\section{Conclusion}

In this paper we have provided a survey on the problem of the early wildfire detection overviewing a number of approaches with the application of WSN.

The existing methods are surveyed and classified into three groups: gas sensing, sensing of environmental parameters, and video monitoring. The analysis of the classification has been aimed at demonstrating the advantages and drawbacks of each of the groups. The gas sensing approach can predict the fire at the stage of pyrolysis emission in the atmosphere. However, due to the high cost and power consumption of these sensor nodes, the WSN deployment on large territories seems unaffordable. The low cost and power consumption along with the simple implementation of the nodes supporting the approach classified as sensing of environmental parameters make the WSN application attractive. Unfortunately, this approach is generally applicable at the open fire stage, which cannot be really helpful for putting out the fire. Video monitoring is useful for the verification of fire detected by conventional temperature and humidity sensors. Video cameras are power 'hungry' devices and without a proper energy scavenging technology application they cannot be used for continuous monitoring purposes.

Summing up we may point out that the gas sensing and video monitoring approaches provide the really early fire 
detection capability. The major restraints for their extensive use remain high power consumption and implementation costs.

Besides, it should be mentioned that only three out of nine WSN platforms presented in this work have been deployed in real conditions. It testifies to the fact that the use of WSNs for wildfire monitoring is still at its initial phase. For a thorough analysis and evaluation of the WSN application, more deployments are required.

Acknowledgement. The author would like to acknowledge Dr Yuliya Verkhovskaya (VNIIEM, Russia) for valuable comments.

\section{References}

[1] Doolin, D.M. and Sitar, N. (2005) Wireless sensors for wildfire monitoring. In Proceedings of Conference on Sensors and Smart Structures Technologies for Civil, Mechanical, and Aerospace Systems, San Diego, CA, USA, 7-10 March 2005, 477-484.

[2] Volokitina, A., Sofronov, M. and Sofronova, T. (2008) Topical scientific and practical issues of wildland fire problem. Mitig. Adapt. Strateg. Glob. Chang. 13(7): 661674.

[3] Fleming, J. and Robertson, R.G. (2003) The Osborne firefinder. In Fire Management TechTips, October 2003, $1-2$.

[4] den Breejen, E., Breuers, M., Cremer, F., Kemp, R., Roos, M., Schutte, K. and DE Vries, J.S. (1998) Autonomous forest fire detection. In Proceedings of the Third International Conference on Forest Fire Research and the Fourteenth Conference on Fire and Forest Meteorology, Luso, Portugal, 16-20 November 1998, 2003-2012.

[5] Leblon, B. (2005) Monitoring forest fire danger with remote sensing. Nat. Hazards 35(3): 343-359.

[6] Burgan, R., Klaver, R. and Klaver, J. (1998) Fuel models and fire potential from satellite and surface observations. Int. J. Wildland Fire 8(3): 159-170.

[7] Chong, C.Y. and Kumar, S.P. (2003) Sensor networks: evolution, opportunities, and challenges. In Proceedings of IEEE 91 (8): 1247-1256

[8] Antoine-Santoni, T., Santucci, J.F., De Gentili, E. and Costa, B. (2008) Wildfire impact on deterministic deployment of a wireless sensor network by a discrete event simulation. In Proceedings of the Fourteenth Mediterranean Electrotechnical Conference, Ajaccio, France, 5-7 May 2008, 204-209.

[9] Moser, C., Thiele, L., Brunelli, D. and Benini, L. (2007) Adaptive power management in energy harvesting systems. In Proceedings of Design, Automation and Test in Europe Conference and Exhibition, Nice, France, 16-20 April, 1-6.

[10] Jiang, X., Taneja, J., Ortiz, J., Tavakoli, A., Dutta, P., Jeong, J., Culler, D. et al. (2007) An architecture for energy management in wireless sensor networks. ACM Sigbed Rev. 4(3): 31-36.

[11] Macil, D., Ageev, A. and Somov, A. (2009) Power consumption reduction in wireless sensor networks through optimal synchronization. In Proceedings of Instru- mentation and Measurement Technology Conference (I2MTC'09), Singapore, 5-7 May 2009, 1346-1351.

[12] Jiang, X., Polastre, J. and Culler, D. (2005) Perpetual environmentally powered sensor networks. In Proceedings of the Fourth International Symposium on Information Processing in Sensor Networks (IPSN'05), Los Angeles, CA, USA, 25-27 April, 463-468.

[13] Martinez, K., Hart, J.K. and ONG, R. (2004) Environmental sensor networks. IEEE Comput. 37(8): 50-56.

[14] Chao, C.M. and Chang, Y.C. (2007) A power-efficient timing synchronization protocol for wireless sensor networks. J. Inf. Sci. Eng. 23(4): 985-997.

[15] IEEE Standards Association (2011), http://standards. ieee.org/about/get/802/802.15.html.

[16] ZigBee specification (2011), www.zigbee.org/Products/ DownloadZigBeeTechnicalDocuments.aspx.

[17] Somov, A., Spirjakin, D., Ivanov, M., Khromushin, I., Passerone, R., Baranov, A. and Savkin, A. (2010) Combustible gases and early fire detection: an autonomous system for wireless sensor networks. In Proceedings of the First International Conference on Energy-Efficient Computing and Networking (e-Energy'10), Passau, Germany, 13-15 April 2010, 85-93.

[18] Roundy, S., Wright, P.K. and Rabaey, J.M. (2004) Energy Scavenging for Wireless Sensor Networks with Special Focus on Vibrations (USA: Kluwer Academic Publishers).

[19] Zhang, P., SAdler, C.M., Lyon, S.A. and Martonosi, M. (2004) Hardware design experience in zebranet. In Proceedings of the Second International Conference on Embedded Networked Sensor Systems (SenSys' 04), Baltimore, Maryland, USA, 3-5 November 2004, 227-238.

[20] Werner-Allen, G., Lorincz, K., Welsh, M., Marcillo, O., Johnson, J., Ruiz, M. and Lees, J. (2006) Deploying a wireless sensor network on an active volcano. IEEE Internet Comput. 10(2): 18-25.

[21] Martinez, K., Hart, J.K. and Ong, R. (2009) Deploying a wireless sensor network in Iceland. In Proceedings of the Third International Conference on Geo Sensor Networks (GSN'09), Oxford, UK, 13-14 July 2009, 131-137.

[22] Selavo, L., Wood, A., Cao, Q., Sookoor, T., Liu, H., Srinivasan, A., Wu, Y. et al. (2007) LUSTER: Wireless sensor network for environmental research. In Proceedings of the Fifth Conference on Embedded Networked Sensor Systems (SenSys'07), Sydney, Australia, 6-9 November 2007, 103-116.

[23] Mainwaring, A., Polastre, J., Szewczyk, R., Culler, D. and Anderson, J. (2002) Wireless sensor networks for habitat monitoring. In proceedings of the First International Workshop on Wireless Sensor Networks and Applications (WSNA' 02), Atlanta, GA, USA, 28 September 2002, 88-97.

[24] Tolle, G., Polastre, J., Szewczyk, R., Culler, D., Turner, N., Tu, K., Burgess, S. et al. (2005) A macroscope in the redwoods. In Proceedings of the Third Conference on Embedded Networked Sensor Systems (SenSys' 05), San Diego, CA, USA, 2-4 November 2005, 5163.

[25] Ho, S., Koushanfar, F., Kosterev, A. and Tittel, F. (2007) LaserSPECks: laser spectroscopic trace-gas sensor 
networks-sensor integration and application. In Proceedings of Sixth International Symposium on Information Processing in Sensor Networks (IPSN'07), Cambridge, MA, USA, 25-27 April 2007, 226-235.

[26] Welsh, E., Fish, W. and Frantz, J. (2003) GNOMES: a testbed for low-power heterogeneous wireless sensor networks. In Proceedings of International Symposium on Circuits and Systems (ISCAS'03), Bangkok, Thailand, 2528 May 2003, 836-839.

[27] Son, B., Her, Y.-S. and Kim, J.-G. (2006) A design and implementation of forest-fires surveillance system based on wireless sensor networks for South Korea mountains. Int. J. Comput. Sci. Netw. Secur. 6(9): 124-130.

[28] TIP50CM mote, MAXFOR Technology (2011), www.maxfor.co.kr.

[29] Bayo, A., Antolin, D., Medrano, N., Calvo, B. and Celma, S. (2010) Development of a wireless sensor network system for early forest fire detection. In Proceedings of European Workshop on Smart Objects: Systems, Technologies and Applications (RFID SysTech' 10), Ciudad, Spain, 15-16 June 2010, 1-7.
[30] Lozano, C. and Rodriguez, O. (2011) Design of forest fire early detection system using wireless sensor networks. J. Electron. Electr. Eng. 3(2): 402-405.

[31] Li, Y., Wang, Z. and Song, Y. (2006) Wireless sensor network design for wildfire monitoring. In Proceedings of the Sixth World Congress on Intelligent Control and Automation, Dalian, China, 21-23 June 2006, 109-113.

[32] Lloret, J., Garcia, M., Bri, D. and Sendra, S. (2009) A wireless sensor network deployment for rural and forest detection and verification. Sensors 9(11): 8722-8747.

[33] Hartung, C., Han, R., Saielstad, C. and Holbrook, S. (2006) FireWxNet: A multi-tiered portable wireless system for monitoring weather conditions in wildland fire environments. In Proceedings of the Fourth International Conference on Mobile Systems, Applications and services (MobiSys' 06), Uppsala, Sweden, 19-22 June 2006, 28-41.

[34] Crossbow Technology (2011), www.xbow.com.

[35] Food and Agriculture Organization of the United Nations (2010) Global forest resources assessment 2010. FAO Forestry Paper 163. 